"This is an Accepted Manuscript of an article published by Taylor \& Francis in MEDICAL TEACHER on December 10 2019, available online:

http://www.tandfonline.com10.1080/0142159X.2019.1687864." 


\section{Clinical Prioritisation Questions: a novel assessment tool to encourage tolerance of uncertainty?}

\section{Clinical Prioritisation Questions in Medicine}

Authors: Sam, Amir H. ${ }^{1}$; Wilson Rebecca K. ${ }^{1}$; Lupton, Martin ${ }^{1}$; Melville, Colin $^{2,3}$; Halse, Omid ${ }^{1}$; Harris, Joanne ${ }^{4}$; Meeran, Karim.

${ }^{1}$ Imperial College School of Medicine,, Imperial College London, London, U.K.

${ }^{2}$ General Medical Council, London, U.K.

${ }^{3}$ Division of Medical Education, University of Manchester. Manchester, U.K.

${ }^{4}$ University of Buckingham, Buckingham, U.K.

Correspondence should be addressed to: Professor Karim Meeran, Department of

Endocrinology, Charing Cross Hospital, Fulham Palace Road, London, W6 8RF.

Email k.meeran@imperial.ac.uk

The authors have informed the journal that they agree that both Amir H Sam and Rebecca K Wilson completed the intellectual and other work typical of the first author. 


\section{Clinical Prioritisation Questions: a novel assessment tool to encourage}

\section{2 tolerance of uncertainty?}

3 Abstract

4 Uncertainty is a common and increasingly acknowledged problem in clinical practice. Current

5 single best answer (SBA) style assessments test areas where there is one correct answer, and

6 as the approach to assessment impacts on the approach to learning, these exams may poorly

7 prepare our future doctors to handle uncertainty. We therefore need to modify our approach to

8 assessment to emphasize reasoning and introduce the possibility of more than one 'correct'

9 answer.

We have developed clinical prioritisation questions (CPQs), a novel formative assessment tool in which students prioritise possible responses in order of likelihood. This assessment format was piloted with a group of medical students and evaluated in comparison with the more traditional single SBA question format in a team-based learning setting.

14 Students reported that they felt ongoing use would help improve their tolerance of uncertainty ( $p<0.01$ ). Furthermore, over $80 \%$ of students felt that CPQs were more reflective of real-life clinical practice. Group based discussions were significantly longer when answering CPQs $(\mathrm{p}<0.01)$, suggesting they may promote richer discourse.

CPQs may have a role in formative assessment to help equip students with the skills to cope with ambiguity and strengthen clinical reasoning and decision-making. Institutions may find them more practical to implement compared with other clinical reasoning assessment tools.

\section{Keywords}




\section{Practice Points}

- CPQs require students to rank possible responses in order of likelihood, with the aim of strengthening clinical reasoning skills and introducing the concept of more than one right answer

- CPQs stimulate longer and potentially richer discourse when used in collaborative learning

- Students felt CPQs were a better representation of how they would approach problems in clinical practice, and ongoing use would improve their preparation for practice.

\section{Notes on Contributors}

Amir H Sam is Director of Assessment at Imperial College School of Medicine, London, U.K.

Rebecca Wilson is a Clinical Education Fellow at Imperial College School of Medicine, London, U.K.

Martin Lupton is the Vice-Dean (Education) for the Faculty of Medicine at Imperial College School of Medicine, London, U.K.

Colin Melville is Medical Director and Director, Education and Standards at the General Medical Council and Hon. Professor of Medical Education at the University of Manchester, Manchester, U.K.

Omid Halse is Deputy Director of Phase 1 at Imperial College School of Medicine, London, U.K.

Joanne Harris is Director of Medical Education, University of Buckingham, U.K.

Karim Meeran is Director of Teaching at Imperial College School of Medicine, London, U.K. 


\section{Introduction}

Physicians are faced with increasingly complex patients, requiring sound clinical reasoning skills to analyse and assess the large volume of information available when making medical decisions. It has been proposed that assessment of clinical reasoning skills should include assessment in the context of uncertainty, where more than one correct answer is plausible (Cooke and Lemay 2017). Furthermore, physicians must be able to tolerate uncertainty in order to provide the best possible care for their patients. It has been shown that doctors who are less tolerant of uncertainty have a higher propensity to order excessive diagnostic tests and institute empirical treatment (Luther and Crandall 2011). This has economic implications as well as placing patients at risk of adverse events. Clinicians who are less comfortable with uncertainty are reluctant to disclose this uncertainty to their patients, and therefore less likely to involve patients in making decisions about their care (Politi and Légaré 2010).

The General Medical Council has made the introduction of the concept of uncertainty in clinical medicine to undergraduates in the United Kingdom a priority, with Outcomes for Graduates (General Medical Council 2018) specifying that 'newly qualified doctors must be able to recognise complexity and uncertainty... and develop confidence in managing these situations.' Medical schools must therefore develop new ways of supporting learners to acknowledge and manage uncertainty, and demonstrate they are meeting these standards.

Assessment and learning are intrinsically linked. Our current medical assessments may give students a misleading view as to the level of certainty in clinical practice; single best answer questions (SBAs) encourage students to focus their learning around a single 'correct' answer to any clinical problem (Epstein 2007). As a result, they may neglect to consider other aspects of the problem, such as differential diagnoses, leading to a narrow scope of learning. Even in Objective Structured Clinical Examinations (OSCEs) the scoring rubrics are often associated 
with a checklist which rewards information gathering, the routine of physical examination and algorithm driven management, rather than clinical reasoning skills (Cooke and Lemay 2017).

In real life, consultations with patients are often complex, with many contextual factors. There are frequently a number of options available regarding approach and management of a clinical case. Importantly, there may be more than one correct approach. Clinicians use clinical reasoning skills alongside patient needs and contextual factors to inform clinical decision making. Introducing new ways of assessing medical students to reward consideration of the likelihood of more than one correct answer -for instance prioritizing a list of diagnostic possibilities- may reward development of clinical reasoning skills and encourage deeper learning (Bowen 2006; Simpkin and Schwartzstein 2016).

Script Concordance Tools (SCTs) have been developed to try and address this problem (Charlin and van der Vleuten 2004). This is where students are assessed by the extent to which their judgements on a clinical case reflect those of a reference 'expert' panel. As the case evolves, more information becomes available to the student, leading to the generation of differential diagnoses or a management plan. Several answers may be considered appropriate; the decisions the student makes are compared to those of the reference panel. However, uptake in the use of these tools has been variable, possibly due to a perception that they are both cost and labour-intensive for faculty to create and deliver to students (Matthieu et al. 2013).

We developed an online formative assessment tool, called Clinical Prioritisation Questions (CPQs), in which students ranked possible diagnoses in order of likelihood, for use in a teambased learning setting. This is similar to the Situational Judgment Test format, which is used in the UK to assess professional behaviours and attitudes for selection to postgraduate medical training (Patterson et al. 2013). The aim of the CPQ assessment format is two-fold; firstly, to encourage clinical reasoning skills through prioritisation of the options, and secondly, to 
97 introduce students to the concept of clinical uncertainty; that there may be more than one correct answer. We set out to evaluate their use in a team-based learning setting and student perceptions of CPQs compared to the more traditional SBAs. This tool was evaluated amongst Year 3 students at our institution. These students had completed the first of three clinical attachments in medicine, surgery or primary care. Students had been exposed to a wide range of teaching formats, including lectures, tutorials, team-based learning and problem-based learning cases. The applied knowledge assessments in Years 1 and 2 typically consisted of multiple-choice questions and short answer questions.

\section{Methods}

All Year 3 medical students at Imperial College School of Medicine were invited to a teambased learning activity as part of their timetabled teaching. There were no other inclusion or exclusion criteria.

Students who attended the team-based learning session were given a short introduction by the session facilitator explaining the rationale for the teaching and introducing the concept of uncertainty in clinical practice. They were then given a total of ten case-based questions; five questions in the CPQ format, and five questions in the SBA format. Firstly, the students answered these questions individually, and then following this they discussed the same cases in teams of 6-8 learners before answering the questions for the second time. They were asked to record the time taken to reach a group consensus for both question formats.

Each case-based question consisted of a clinical scenario (which included the presentation, examination findings and investigation results, as necessary) and a lead-in question. For the SBA question format, students were asked to select the best answer from five options. For the CPQ format, students were asked to rank the five options from most likely to least likely (figure 
1). The question topics were mapped to the curriculum to ensure alignment with the learning objectives for Year 3. [Figure 1 near here]

The exercise was conducted on iPad tablets using the online assessment software Practique (Fry-It Ltd, London, UK). All answers were entirely machine marked using the examination software. CPQs were marked using the same marking matrix that is used for situational judgement tests, where up to 20 marks per question are available (Patterson et al. 2013). This marking matrix was used as both faculty and students are familiar with it as means of rewarding more than one answer. For each of the five response options up to four marks are available, with marks deducted according to how far away the option was placed relative to the correct answer. The minimum score on any question is therefore $8 / 20$, and random guessing scores on average $12 / 20$. The 'best' order was determined by a group of three clinicians for each question.

Following completion of the team-based learning activities, the students were shown an example of CPQ responses from a previous session. The aim of this was to illustrate that uncertainty is a routine part of clinical practice and there is often disagreement amongst medical professionals as to the best answer. Figure 2 shows the variation in responses to a CPQ question on an individual basis compared to a group basis. [Figure 2 near here]

During the teaching session, students were asked to complete a survey regarding their feelings around uncertainty in medicine and their perceptions of CPQs and SBAs. This was carried out using a cloud-based web tool (Mentimeter), to which the students could anonymously submit their responses via their iPad (figure 3). [Figure 3 near here]. This consisted of a series of Likert items to assess student response to uncertainty when answering both question formats; these were adapted from the Physicians Response to Uncertainty scale (Gerrity et al. 1995). It also comprised of some binary questions regarding student perceptions of both question formats. 
144 Outcomes measured were (1) individual and group scores for both formats, (2) time taken to 145 reach a consensus in group discussion (3) response to uncertainty in both formats using a Likert scale (4) student opinions regarding both formats using binary questions.

147 Statistical analyses were performed using IBM SPSS Statistics for Windows Version 25.0 148 (IBM Corp, Armonk, NY, USA) and PRISM Version 5.0C (Graphpad Software, Inc., San 149 Diego, CA, USA). The Kolmogorov-Smirnov test was used to assess normal distribution of the data; all data was non-parametric. Wilcoxon signed rank test was used to examine the difference in time to reach a group consensus between the CPQ and the SBA question formats. McNemar's test was used to compare the responses to the binary questions regarding students' opinion of CPQs and SBAs.

\section{Results}

There were 302 students enrolled in Year 3. A total of 245 students participated in the teaching session. 234 of these completed the course survey.

Scores for both the CPQs and the SBAs improved following group discussion. Median individual CPQ score was $67 \%$ compared to a median group CPQ score of $78 \%$. Median individual SBA score was $40 \%$ compared to a median group SBA score of $60 \%$.

161 Group discussion during the TBL activity was significantly longer for CPQ questions compared to SBA questions. The median length of discussion time to achieve a consensus answer on 5 questions was 11 minutes 42 seconds for the CPQs compared to 7 minutes 48 seconds for the SBAs $(\mathrm{p}<0.01)$.

Students were asked five separate questions regarding their response to uncertainty when answering CPQs compared to SBAs using a Likert scale response. On all five questions a 
higher proportion of students reported feeling more anxious when answering CPQs compared to SBAs (figure $4 a$ and $4 b$ ). Significantly more students felt that continued use of CPQs would improve their tolerance of uncertainty compared to continued use of SBAs $(66 \%(n=154)$ vs $42 \%(n=98), p<0.01) .80 \%(n=187)$ of students felt that CPQs were a better representation of how they would be expected to answer questions in clinical practice. $60 \%(n=140)$ felt that SBAs were easier than CPQs, and 56\% $(n=132)$ felt CPQs would change their learning and revision strategy. Furthermore, $62 \%(n=144)$ felt that continued use of CPQs would improve their preparation for practice as a junior doctor. [Figures $4 \mathrm{a}$ and $4 \mathrm{~b}$ near here]

The free-text single word responses were grouped into broad themes to identify what was most frequently cited. Of these, the three themes most commonly expressed were:

(1) CPQs are difficult for me e.g. challenging, difficult, hard

(2) CPQs are good for me e.g. good, great, fun

(3) CPQs are interesting e.g. different, interesting, thought-provoking

\section{Discussion}

This pilot study set out to compare CPQs, an assessment tool in which students prioritise a list of five options in order of likelihood, with more traditional SBA questions, in a TBL setting. The UK General Medical Council has specified the ability to propose prioritised differential diagnoses as an outcome for newly qualified doctors (General Medical Council 2018). Asking students to rank answers in order of likelihood may help them develop clinical reasoning skills as they are required to apply, analyse and synthesise the information in the vignette to prioritise the options. In doing so they can also acknowledge the inherent uncertainty within the context of the case. As CPQs reward consideration of differential diagnoses, they will have a less 
definitive 'right' answer than an SBA. In an SBA the possible scores are 0 and 1 (reinforcing a notion of 'black and white'), whereas in a CPQ the students may score a range of marks, introducing the notion of 'shades of grey'. Students felt that ongoing use of CPQs in the medical school curriculum would assist them in developing tolerance of uncertainty and in clinical practice.

We are unable to directly compare the scores attained between the CPQ and SBA formats due to the differences in which they are marked. With both the CPQ and SBA question formats, students scored more highly following group discussion. However, this improvement may partially be accounted for by the opportunity to have a 'second look' at the questions. The CPQs stimulated a lengthier debate than SBAs; we speculate that the uncertainty they generate encourages richer discourse amongst students (Schwartzstein and Roberts 2017), and therefore they may help to drive deeper learning.

Non parametric tests were used in our statistical analysis as our data was not normally distributed; however, it must be acknowledged that small differences may be statistically significant due to the sample size. The number of items used in this pilot study was small; we are therefore unable to draw any conclusions regarding reliability of CPQs. Further work is needed to assess the other aspects of utility of CPQs, (van der Vleuten 1996) including their validity, cost effectiveness, acceptability and educational impact. It may also be useful to assess correlation with SCTs, and other more widely used examination formats such as SBAs and OSCEs. Ideally, the next step would be to administer CPQs as a formative assessment tool to a cohort over the course of their studies and evaluate the differences in their scores and tolerance of uncertainty as they progress from novice to expert.

\section{Conclusion}


214 Our results suggest that CPQs may help equip students with the skills to cope with uncertainty,

215 and help strengthen clinical reasoning and decision-making, through prioritising a list of

216 diagnostic possibilities. They appear to stimulate richer case-based discussions and students

217 find them acceptable and relevant. We propose they may have a role to play as a formative

218 assessment tool, but further work is needed to formally assess their utility. Institutions may

219 find them more practical to implement compared with other clinical reasoning assessment 220 tools.

221

222

223 
224 Acknowledgements and disclosures

225

226 Acknowledgements

227 The authors wish to thank Keean Schupke at FRY-IT for his help with the Practique

228 software.

229 Funding/Support

230 None.

231 Other disclosures

232 None.

233 Ethical Approval

234 This study was granted ethical approval by the Medical Education Ethics Committee,

235 Imperial College London.

236 Disclaimer

237 None.

238 Previous presentations

239 None.

240 


\section{References}

1. Bowen JL. 2006. Educational strategies to promote clinical diagnostic reasoning. $\mathrm{N}$ Engl J Med. 355(21):2217-25.

2. Charlin B, van der Vleuten C. 2004. Standardized assessment of reasoning in contexts of uncertainty: the script concordance approach. Eval Health Prof. 27(3):304-19.

3. Cooke S, Lemay J-F. 2017. Transforming medical assessment: integrating uncertainty into the evaluation of clinical reasoning in medical education. Acad Med. 92(6):74651.

4. Epstein RM. 2007. Assessment in Medical Education. N Engl J Med. 356(4):387-96.

5. General Medical Council. 2018. Outcomes for Graduates 2018. London (UK): General Medical Council.

6. Gerrity MS, White KP, DeVellis RF, Dittus RS. 1995. Physicians' reactions to uncertainty: Refining the constructs and scales. Motivation and Emotion. 19:175-91

7. Luther VP, Crandall SJ. 2011. Ambiguity and uncertainty: neglected elements of medical education curricula? Acad Med. 86(7):799-800.

8. Mathieu S, Couderc M, Glace B, Tournadre A, Malochet-Guinamand S, Pereira B, Dubost JJ, Soubrier M. Construction and utilization of a script concordance test as an assessment tool for DCEM3 (5 $5^{\text {th }}$ year) medical students in rheumatology. 2013. BMC Medical Education. 13:166.

9. Patterson F, Ashworth V, Good D. 2013. Situational judgement tests: A guide for applicants to the UK Foundation Programme. London (UK); Medical Schools Council. [accessed 24 ${ }^{\text {th }}$ April 2019] https://isfporguk.files.wordpress.com/2017/04/situationaljudgement-tests-monograph.pdf

10. Politi MC, Légaré F. 2010. Physicians' reactions to uncertainty in the context of shared decision making. Patient Educ Couns. 80(2):155-7. 
266

267

268

269

270

271

272

11. Schwartzstein RM, Roberts DH. 2017. Saying goodbye to lectures in medical schoolparadigm shift or passing fad? N Engl J Med. 377(7):605-7.

12. Simpkin AL, Schwartzstein RM. 2016. Tolerating uncertainty - the next medical revolution? N Engl J Med. 375(18):1713-5.

13. Van der Vleuten CPM. The Assessment of Professional Competence: Developments, Research and Practical Implications. 1996. Adv Health Sci Educ. 1:41-67.

2 
Figure 1: an example of a $C P Q$ and an SBA for respiratory medicine.

Example CPQ:

A 50 year old Afro Caribbean man has a 4 month history of a dry cough and increasing breathlessness. He has not lost any weight. He has also noticed a rash on his legs. On examination, both ankles are swollen and there are tender round lesions on both shins. His temperature is $36.8^{\circ} \mathrm{C}$, pulse rate $72 \mathrm{bpm}, \mathrm{BP} 128 / 65 \mathrm{mmHg}$ and oxygen saturations $95 \%$ breathing air.

Investigations: serum calcium $2.8 \mathrm{mmol} / \mathrm{L}, \mathrm{ESR} 48 \mathrm{~mm} / \mathrm{h}$

Chest X-ray: widened mediastinum

Rank the following diagnoses, with 1 being the most likely and 5 being the least likely.

A. Idiopathic pulmonary fibrosis (4)

B. Sarcoidosis (1)

C. Systemic lupus erythematosus (5)

D. Tuberculosis (2)

E. Bronchogenic carcinoma (3)

Example SBA:

A 55 year old man has a 3 month history of a cough productive of green sputum. He has noticed he is becoming short of breath when walking his dog and has increasing fatigue. $\mathrm{He}$ has not lost any weight. He is an ex-smoker with a 40 pack year history. His BP is $135 / 76 \mathrm{mmHg}$, respiratory rate 18 breaths per minute and oxygen saturation $94 \%$ breathing air. There is wheeze bilaterally and bibasal crackles on auscultation of the chest.

Investigations:

Chest X-ray: hyperinflated lung fields

What is the most appropriate investigation to confirm the diagnosis?
A. Bronchoscopy
B. CT thorax
C. Peak expiratory flow rate
D. Spirometry
E. Sputum culture 
Figure 2: an example of the variation in responses to a CPQ on an individual basis and following a group discussion.

Q3. A 19 year old university student with no past medical history presents with headache and neck stiffness. Lumbar puncture microscopy shows 3800 white cells, $90 \%$ of which are neutrophils. Rank the following organisms which may have caused this presentation with 1 being the most likely and 5 being the least likely.

Individual responses:

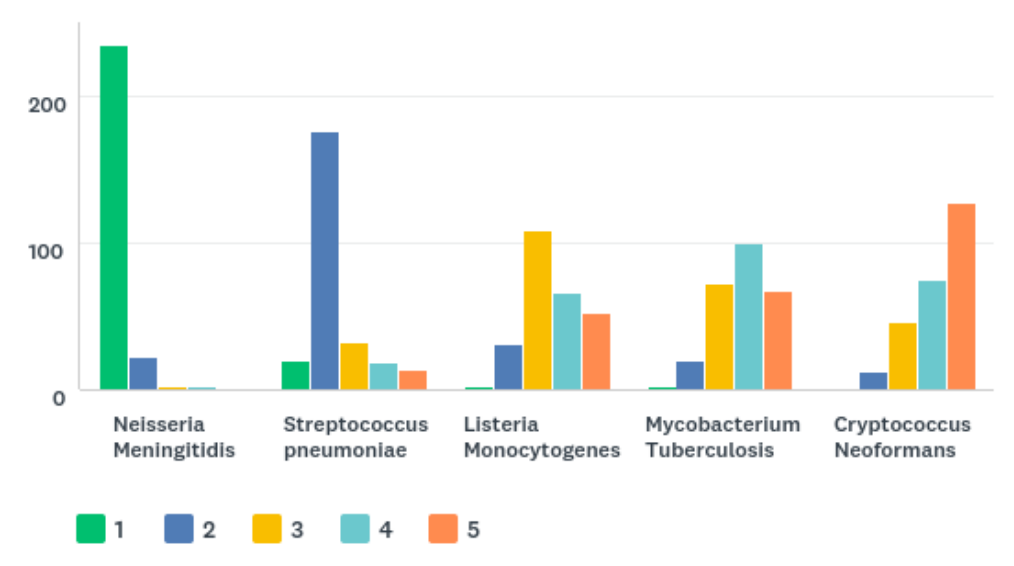

Team responses:

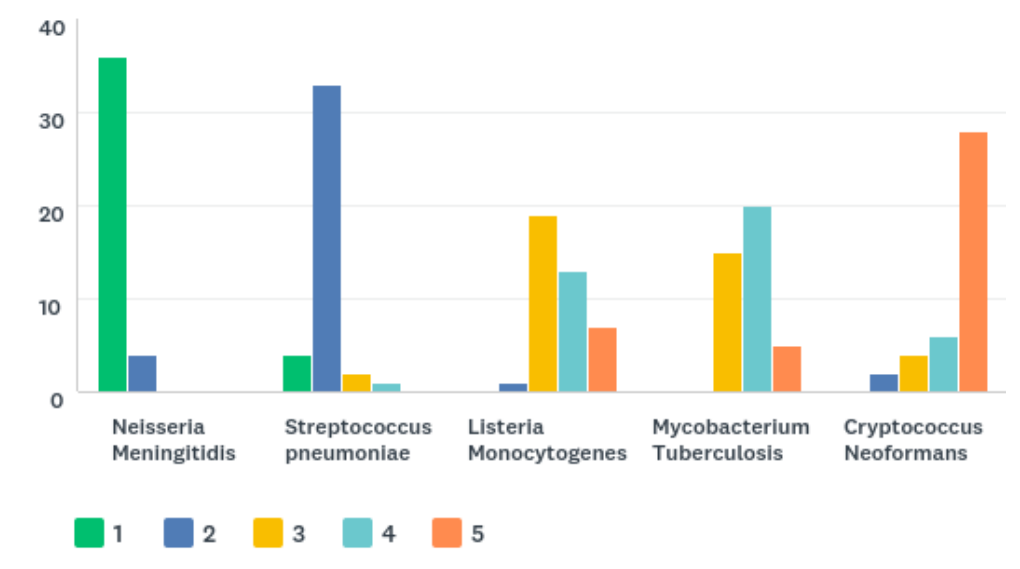


Figure 3: Survey given to students. Questions 1-5 were adapted from the Physicians Response to Uncertainty scale (Gerrity et al. 1995).

Students were asked to rate the following 5 statements on a 5-point Likert scale (strongly disagree, disagree, neutral, agree, and strongly agree) in relation to both CPQs and SBAs:

1. I felt anxious answering the questions as I was unsure of the diagnosis

2. I find the uncertainty involved in the questions disconcerting

3. Uncertainty in the questions makes me uneasy

4. I am quite comfortable with the uncertainty in the questions

5. The uncertainty of the questions troubles me.

Secondly, they were asked to select one of two statements to the following questions:

6. Do you think your tolerance of uncertainty would change by answering SBAs?

a. Yes, I would tolerate uncertainty better

b. No, I would not be any better at tolerating uncertainty

7. Do you think your tolerance of uncertainty would change by answering CPQs?

a. Yes, I would tolerate uncertainty better

b. No, I would not be any better at tolerating uncertainty

Thirdly, the students were asked to rate the following statements on a 5-point Likert Scale (strongly disagree, disagree, neutral, agree, strongly agree):

8. Questions in an SBA format are easier than those in a CPQ format

9. CPQs are a better representation of how I would be expected to answer questions in clinical practice

10. Having exams in a CPQ format would change my learning and revision strategy

11. Using CPQs in assessments would improve my preparation for practice.

Finally, the students were asked to give three words to describe their feelings about CPQs in a free text box. 


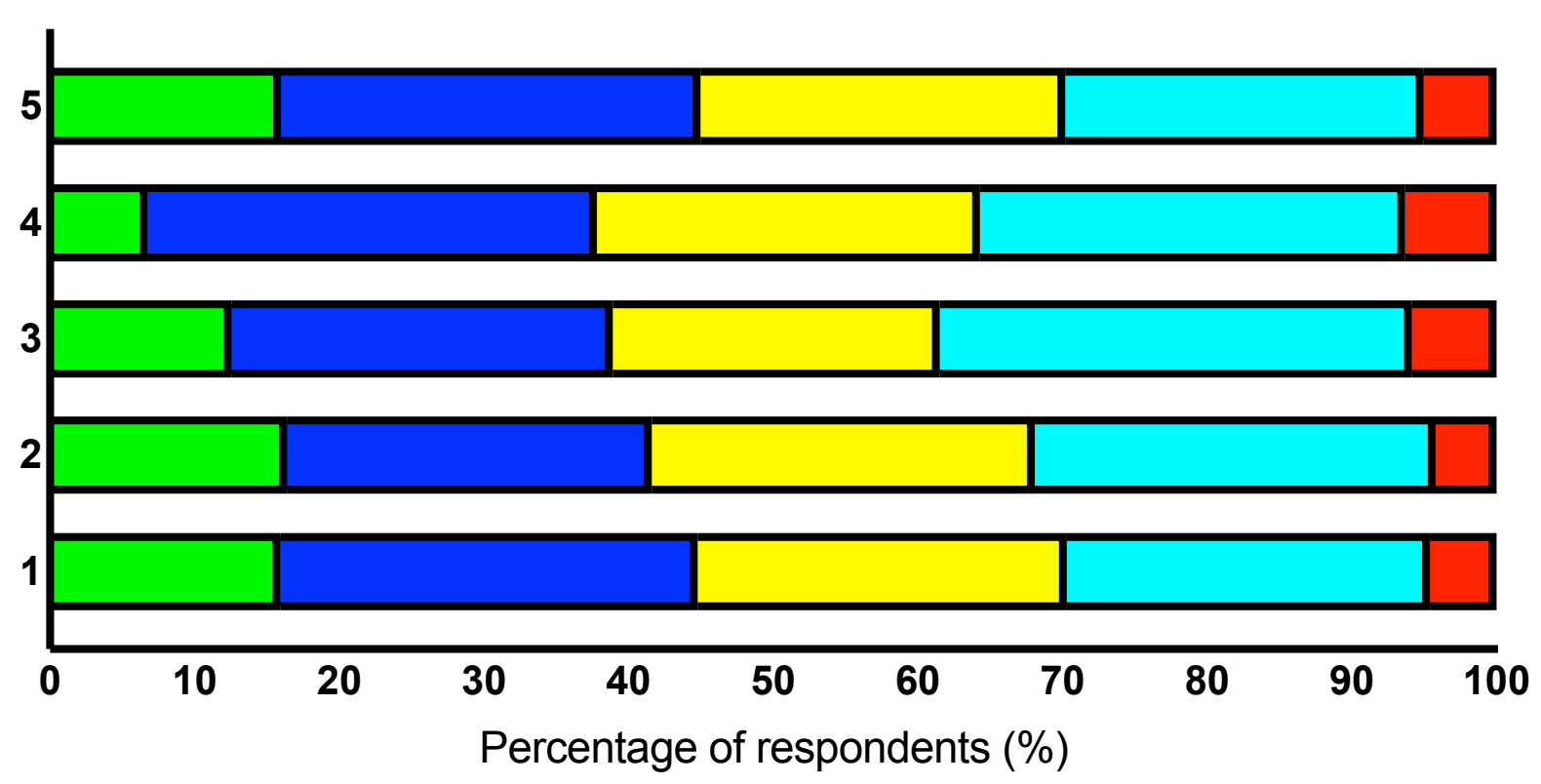

$\square$ Strongly agree

$\square$ Agree

$\square$ Neutral

$\square$ Disagree

$\square$ Strongly disagree

Figure 4a The percentage of responders selecting each point on a 5-point Likert scale are shown for each of five statements about single best answer questions (SBAs). 1. I felt anxious answering the questions as I was unsure of the diagnosis. 2. I find the uncertainty involved in the questions disconcerting. 3. Uncertainty in the questions makes me uneasy. 4. I am quite comfortable with the uncertainty in the questions (reverse scored). 5 . The uncertainty of the questions troubles me. 
